\title{
Contenido de $\beta$-Caroteno, Hierro y Zinc, Efecto de Almacena- miento y Tipo de Cocción en Genotipos de Camote (Ipomoea batatas L)
}

\author{
B-Carotene, Iron and Zinc Content, Effect of Storage and Type of Cooking in \\ Sweet Potato (Ipomoea batatas L) Genotypes
}

\author{
Rossemary V. Carpio R. ${ }^{1,3}$, Alejandrina Sotelo M. ${ }^{2}$, Wolfgang J. Grüneberg ${ }^{1}$
}

\section{Resumen}

\begin{abstract}
El camote (Ipomoea batatas L) es el principal componente en la dieta de millones de personas que viven en países tropicales, y un mejoramiento en el contenido de $\beta$ caroteno podría incrementar el suministro de vitamina A para satisfacer la mayor parte de las necesidades diarias de la gente. En el presente estudio, el contenido de $\beta$-caroteno $(\mathrm{BC})$, hierro $(\mathrm{Fe})$ y zinc $(\mathrm{Zn})$ fueron determinados en 25 genotipos de camote provenientes de las localidades de La Molina y San Ramón (Perú). Así mismo, los cambios en el contenido de $\mathrm{BC}$ en siete genotipos de camote fueron investigados usando diferentes métodos de cocción y tiempos de almacenamiento. El contenido de $\mathrm{BC}$ fue medido vía absorbancia a 450 nm e identificado a través del HPLC y el contenido de Fe y Zn fueron determinados a través de técnicas de ICP-OES. Los resultados muestran variación significativa entre genotipo, ambiente y en la interacción genotipo-ambiente. La concentración de $\mathrm{BC}$ en las raíces de camote recién cosechado varió entre 7.62 y $18.93 \mathrm{mg} / 100 \mathrm{~g}$ en peso fresco (PF). El contenido de hierro presentó un rango de $0.40-0.96 \mathrm{mg} / 100 \mathrm{~g} \mathrm{PF}$ y el contenido de zinc presentó un rango de $0.25-0.51 \mathrm{mg} / 100 \mathrm{mg}$ PF. Los genotipos 440442 y 440518 presentaron los niveles más altos de BC en los dos ambientes. Diferencias en el contenido de $\mathrm{BC}$ fueron encontrados entre los métodos de cocción y los tiempos de almacenamiento. La concentración de $\mathrm{BC}$ de las raíces cocidas por ebullición $(15.15 \mathrm{mg} / 100 \mathrm{~g} \mathrm{PF})$ fue mayor que las raíces cocidas por horneado $(14.29 \mathrm{mg} / 100 \mathrm{~g} \mathrm{PF})$; sin embargo, no hubo diferencias entre los genotipos 440442 y 420081 por efecto del método de cocción. Asimismo, el contenido de $\mathrm{BC}$ se incrementó durante el tiempo de almacenamiento, donde el genotipo 440442 presentó los mayores niveles de $\mathrm{BC}$ durante el tiempo de almacenamiento, mientras que los genotipos 440413 y 440513 no presentaron cambios significativos.
\end{abstract}

Palabras clave: camote, $\beta$-caroteno, hierro, zinc, cocción, almacenamiento

\footnotetext{
${ }^{1}$ División de Genética, Genómica y Mejoramiento de Cultivos (GGCI), Centro Internacional de la Papa (CIP), Lima, Perú

${ }^{2}$ Facultad de Zootecnia, Departamento de Nutrición, Universidad Nacional Agraria La Molina (UNALM), Lima, Perú

${ }^{3}$ E-mail: r.carpio@cgiar.org
} 
Sweet potato (Ipomoea batatas L) is the main component in the diet of millions of people living in tropical countries, and an improvement in $\beta$-carotene content could increase the supply of vitamin A to meet a greater part of the daily requirements of people. In the present study, the content of $\beta$-carotene $(\mathrm{BC})$, iron $(\mathrm{Fe})$ and zinc $(\mathrm{Zn})$ were determined in 25 genotypes of sweet potato from two localities: La Molina and San Ramón. Furthermore, the changes in $\mathrm{BC}$ content in seven sweet potato genotypes were investigated using different cooking methods and storage times. The content of $\mathrm{BC}$ was measured via absorbance at $450 \mathrm{~nm}$ and identified through HPLC while Fe and Zn content were determined through ICP-OES techniques. The results show significant variation between genotype, environment and in the genotype-environment interaction. $\mathrm{BC}$ content in freshly harvested raw root ranged from 7.62 to $18.93 \mathrm{mg} / 100 \mathrm{~g}$ in fresh weight (PF), iron content ranged from 0.40 to $0.96 \mathrm{mg} / 100 \mathrm{~g} \mathrm{PF}$ and the zinc content ranged from 0.25 to 0.51 $\mathrm{mg} / 100 \mathrm{mg}$ PF. Genotypes 440442 and 440518 showed the highest levels of BC in both localities. Differences in $\mathrm{BC}$ content were found among cooking methods and storage times. BC concentration in boiled roots $(15.15 \mathrm{mg} / 100 \mathrm{~g} \mathrm{PF})$ evaluated was higher than in baked roots ( $14.29 \mathrm{mg} / 100 \mathrm{~g} \mathrm{PF}$ ); however, without differences between genotypes 440442 and 420081 due to the cooking methods. BC content of root increased with storage time, where genotype 440442 showed the highest levels of $\mathrm{BC}$ during storage time, while genotypes 440413 and 440513 did not differ during storage time.

Key words: sweet potato, $\beta$-carotene, iron, zinc, cooking, storage

\section{INTRODUCCIÓN}

Se han alcanzado extraordinarios logros con el camote de pulpa anaranjada (Ipomea batatas L) en zonas empobrecidas del África, donde la carencia de vitamina A condena a millones de niños a la ceguera (Tiempo Agrario, 2010). El camote es un cultivo que provee importantes cantidades de carbohidratos, fibra y muchos micronutrientes como el hierro y zinc (Woolfe, 1992). Sin embargo, las variedades de pulpa anaranjada tienen características aún más resaltantes ya que presentan importantes cantidades de $\beta$ caroteno, el cual es un precursor de la vitamina A (Andrade et al., 2009). El $\beta$-caroteno es la más abundante provitamina A en los alimentos. Entre el 10 al $50 \%$ de $\beta$-caroteno consumido es absorbido en el tracto intestinal, siendo convertida en vitamina A en la pared intestinal. La eficiencia de la absorción del $\beta$-caroteno disminuye a medida que aumenta la ingesta; asimismo, la conversión de vitamina A es regulada por el estatus de la vitamina A en el individuo. Durante el procesamiento de alimentos, el calor, aire y luz son las principales causas naturales que generan la descomposición de los carotenoides (Wang, 1994, citado por García-Casal et al., 1998).

Los factores ambientales también juegan un rol importante, ya que influyen en la calidad y el contenido de nutrientes del cultivo; además, existe la probabilidad de que estos interactúen con factores genéticos (Bonierbale et al., 2010). Esta situación hace que las variedades respondan de manera diferente ante un determinado ambiente, siendo afectados los metabolitos secundarios (carotenoides y minerales). La información sobre la cuantificación del contenido de $\beta$ caroteno en genotipos de camote desarrollados en lugares diferentes, permite mejorar la cantidad de estos compuestos a través de estudios de caracterización y mejoramiento genético, o de manejo agronómico. Además del ambiente, otros factores poscosecha, como 
el almacenamiento y procesamiento, también afectan el contenido de los nutrientes en el camote (Bechoff et al., 2011).

Del mismo nivel de relevancia, los minerales como el hierro y zinc son micronutrientes de importancia, que permiten producir enzimas, hormonas y otras sustancias esenciales que promueven el crecimiento y desarrollo del organismo. Asimismo, su ausencia podría traer consecuencias severas para el individuo (FAO/WHO, 2002). Los minerales en los alimentos también se encuentran influenciados por las propiedades del suelo, interacción de nutrientes y la parte de la planta (Moraghan y Mascagni, 1991).

Por lo expuesto, en el presente estudio se plantearon los siguientes objetivos: determinar el contenido de $\beta$-caroteno, hierro y zinc en 25 genotipos de camote de pulpa anaranjada procedentes de las localidades de Lima y San Ramón, Perú, y evaluar el efecto del almacenamiento y tipos de cocción sobre el contenido de $\beta$-caroteno en siete genotipos selectos.

\section{Materiales y MéTodos}

\section{Material Vegetal}

Veinticinco genotipos mejorados de camote (Ipomoea batatas L.) provenientes del Banco de Germoplasma del Centro Internacional de la Papa (CIP), fueron designados para este ensayo. Los genotipos fueron sembrados en la estación experimental de San Ramón (800 msnm, Junín, Perú) y en la estación experimental de La Molina (241 msnm, Lima, Perú), ambas estaciones pertenecientes al CIP. La temperatura mínima y máxima promedio en San Ramón fue de 18.9 y $32.5^{\circ} \mathrm{C}$ con una precipitación promedio anual de $2000 \mathrm{~mm}$ (Reynel y León, 1989); en tanto que en La Molina fueron de $14.6 \mathrm{y}$ $28.7^{\circ} \mathrm{C}$, respectivamente, con una precipitación promedio anual de $60 \mathrm{~mm}$ (INIA, 2016).
La primera siembra (25 genotipos) fue en septiembre de 2009 y la segunda siembra (genotipos selectos) en enero y febrero de 2010, tanto en La Molina como en San Ramón. Para la siembra, se utilizó un diseño de bloques completamente al azar, con dos repeticiones y 10 plantas por parcela. La distancia entre surcos fue $0.9 \mathrm{~m}$ y entre plantas de $0.25 \mathrm{~m}$. El área total del campo fue de $187.2 \mathrm{~m}^{2}$. Se utilizó un fertilizante a base de nitrógeno, fósforo y potasio (20:20:20) durante la siembra, donde las dosis estuvo basada en un análisis de suelo para ambas localidades.

La cosecha se realizó a los 140 días de la siembra. En ese momento se procedió con el muestreo de las raíces de camote, siguiendo el procedimiento propuesto por Porras et al. (2014). Las muestras identificadas por genotipo y repetición fueron trasladas vía terrestre al laboratorio después de un día de la cosecha y almacenadas a $5{ }^{\circ} \mathrm{C}$ por tres días en un ambiente oscuro, bien ventilado y protegido del polvo hasta su posterior preparación.

\section{Distribución del material vegetal}

Para la primera parte de la investigación (caracterización de los 25 genotipos), se tomó una muestra representativa de 4-5 raíces de tamaño uniforme, colectadas en forma aleatoria de 10 plantas por cada repetición, genotipo y localidad, para la determinación del contenido de $\beta$-caroteno, hierro y zinc. Para la segunda parte de la investigación (efecto de cocción y almacenamiento) se emplearon siete genotipos seleccionados de la primera parte del trabajo, según el contenido de $\beta$-caroteno (alto, medio y bajo) y fueron sembrados en las dos localidades.

Se evaluaron las características físicoquímicas de las raíces resultantes. La distribución de los tratamientos se presenta en la Figura 1. La preparación de muestras y los análisis fisicoquímicos se realizaron en el Laboratorio de Calidad y Nutrición ubicado en la Estación Experimental de la Molina. 
$1^{\text {era }}$ parte

Se determinó:

B-caroteno, hierro y zinc
Material vegetal

Genotipos de camote crudo

(25 genotipos)

\begin{tabular}{lll} 
& La Molina & San Ramón \\
\hline & La Molina & San Ramón \\
$2^{\text {da }}$ parte & Cocción & Almacenamiento \\
Se determinó: & (7 genotipos) & (7 genotipos) \\
B-caroteno y materia seca & Crudo & 2,6 y 9 semanas \\
& Ebullido & \\
& Horneado
\end{tabular}

Figura 1. Distribución del material vegetal para los análisis de las muestras crudas, cocidas y almacenadas

\section{Cocción}

Se tomó una muestra representativa de 21 raíces de tamaño uniforme colectadas en forma aleatoria de 10 plantas por cada repetición y genotipo, y se distribuyeron para su estudio en tres tipos de cocción (crudo, ebullición y horneado).

\section{Almacenamiento}

Se tomó una muestra representativa de 30 raíces de tamaño uniforme colectadas en forma aleatoria de 10 plantas por cada repetición, genotipo y localidad. Las muestras fueron distribuidas para su estudio en tres tiempos de almacenamiento (2, 6 y 9 semanas).

\section{Preparación de Muestra}

\section{Raíces recién cosechadas}

Las raíces fueron lavadas con abundante agua, enjuagadas con agua desionizada y secadas con papel toalla. Posteriormente, se pelaron y cortaron en forma longitudinal en

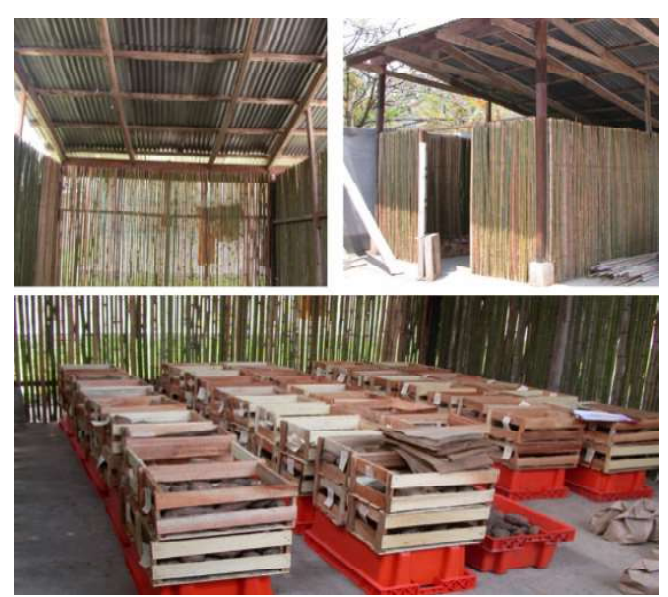

Figura 2. Almacenamiento de raíces de camote en la localidad de San Ramón

cuatro secciones. Una muestra homogénea de $50 \mathrm{~g}$ fue obtenida de los lados opuestos de la raíz. Las muestras fueron colocadas en bolsas de polipropileno, liofilizadas a $27^{\circ} \mathrm{C}$ por $72 \mathrm{~h}$, molida usando molinos de martillo y almacenadas en cámara de frío a $-20^{\circ} \mathrm{C}$ (Porras et al., 2014) para el análisis de $\beta$ caroteno. 
Para el análisis de minerales, se tomó una muestra representativa y homogénea de $50 \mathrm{~g}$ de las otras dos secciones restantes de cada raíz y se colocó en placa Petri. Las muestras fueron secadas en estufa a $180{ }^{\circ} \mathrm{C}$ durante $24 \mathrm{~h}$. Posteriormente, fueron molidas y almacenadas en bolsas de polietileno a temperatura ambiente hasta el análisis de minerales.

\section{Cocción de las raíces}

- Por ebullición: Las raíces crudas sin pelar fueron colocadas en una olla de acero inoxidable y se cubrieron con agua destilada (1.5-2.0 L) y se hirvieron a $100{ }^{\circ} \mathrm{C}$, manteniendo la olla tapada. La cocción se mantuvo por 35 a 40 min donde las raíces quedaron completamente cocidas. La cocción se dio por concluida cuando se pudo introducir fácilmente la punta de un tenedor en el centro de la raíz. Luego de la cocción, se dejó en reposo durante $10 \mathrm{~min}$ para luego remover manualmente la cáscara utilizando un cuchillo. Las raíces sin cascara fueron divididas longitudinalmente en cuatro secciones; se tomó una muestra de $100 \mathrm{~g}$ de los lados opuestos, se guardaron en bolsas de polietileno y se almacenaron a $-20^{\circ} \mathrm{C}$ por $24 \mathrm{~h}$. Luego fueron liofilizadas a $27^{\circ} \mathrm{C}$ por $72 \mathrm{~h}$ y molidas en un molido de martillo. Finalmente, fueron almacenadas a $-20{ }^{\circ} \mathrm{C}$ para el análisis de $\beta$ caroteno.

- Por horneado: Las raíces crudas fueron envueltas en papel aluminio y colocadas uniformemente en una bandeja de acero inoxidable, ubicada en el centro del horno de una cocina a gas. La temperatura y tiempo de cocción se fijaron a $200^{\circ} \mathrm{C}$ y $60 \mathrm{~min}$, respectivamente. Estos parámetros fueron establecidos según pruebas preliminares. Después de la cocción, las raíces se enfriaron por 15 min y se continuó con el procedimiento utilizado para las muestras cocidas por ebullición.

\section{Almacenamiento de las raices}

Treinta raíces por repetición y genotipo en la localidad de San Ramón fueron colocadas en cajas de madera, las cuales estuvieron encima de jabas de plástico para evitar el ingreso de roedores. Las cajas fueron almacenadas en un ambiente bien ventilado construido con bambú (Figura 2). Las condiciones del almacenamiento no fueron controladas; sin embargo, durante el tiempo del almacenamiento se registraron en el almacén temperaturas entre 15 y $26{ }^{\circ} \mathrm{C}$ y humedad entre 44.0 y $89.4 \%$. En el transcurso del almacenamiento se fueron descartando las raíces que presentaban pudrición o algún otro deterioro. Posteriormente, 7-8 raíces por cada repetición y genotipo fueron colectadas por periodo de almacenamiento y trasladada por vía terrestre al laboratorio de La Molina. Al día siguiente, se tomaron las muestras de laboratorio, siguiendo los procedimientos indicados anteriormente para las raíces recién cosechadas.

\section{Métodos Analíticos}

Los carotenoides totales y el $\beta$-caroteno fueron determinados mediante técnicas de espectrofotometría (UV-Visible) a $450 \mathrm{mn}$ y cromatografía (HPLC), según los métodos propuestos por Rodríguez y Kimura (2004). La determinación del contenido de hierro y zinc se realizó a través de técnicas de ICPOES, según Hansen et al. (2009).

\section{Análisis Estadístico}

Los datos fueron sometidos a la prueba de Shapiro y Wilk (1965) para determinar si cumplen con una distribución normal. Las variables (x) que no se distribuyeron normalmente fueron sometidas a una transformación escalar $\mathrm{Y}=\log 10(\mathrm{x})$.

Se realizó un análisis de varianza simple (ANOVA) por cada localidad, considerando un diseño de bloques completamente al azar con dos repeticiones (las repeticiones provienen de campo). Los promedios 
genotípicos fueron comparados por la prueba de Tukey. El efecto de ambiente ( $\mathrm{La}$ Molina y San Ramón) y la interacción genotipo $\mathrm{x}$ ambiente fue analizado por ANOVA considerando los genotipos como fijos y las localidades como efecto aleatorio.

El efecto de los métodos de cocción y almacenamiento fueron analizados por ANOVA, considerando a los genotipos y tratamientos de cocción (crudo, hervido y horneado) y almacenamiento (2, 6 y 9 semanas) como efectos fijos, y los promedios se compararon mediante la prueba de Tukey.

Los estudios de interacción genotipoambiente fueron realizados mediante los modelos AMMI (Additive Main effects and Multiplicative Interaction). En los análisis estadísticos se utilizó el software R.

\section{Resultados y Discusión}

El contenido de materia seca, $\beta$-caroteno, hierro y zinc en 25 genotipos de camote sembrados en las localidades de La Molina y San Ramón se muestra en el Cuadro 1. Los resultados se encuentran expresados en peso fresco (PF).

\section{Materia Seca}

El contenido de materia seca (MS) en los genotipos sembrados en La Molina y San Ramón presentó un rango de 18 a 31\% y de 21 a $41 \%$, respectivamente, con diferencias significativas entre genotipos y sin diferencias significativas en la interacción genotipoambiente. La prueba de Tukey mostró que los genotipos 440060, 401526 y 440513 presentaron los valores más altos de materia seca (\%MS) en ambas localidades $(34.07,31.89 \mathrm{y}$ $31.74 \%$, respectivamente). Estos resultados fueron similares a los reportados por Kapinga et al. (2007), quienes obtuvieron valores entre 31 a $35 \%$ en un grupo de camotes de pulpa anaranjada procedentes de la región SubSahariana. Por su parte, Tumwegamire (2011) señala valores mayores o iguales a $29.5 \%$ en cinco variedades de camote de pulpa anaranjada. El mayor valor de MS en las raíces provenientes de San Ramón podría ser consecuencia del clima que presenta San Ramón (ambiente cálido con pocas precipitaciones, presencia de sequías y baja fertilidad en el suelo).

\section{$\beta$-caroteno}

Se encontró diferencia significativa $(p<0.05)$ en el contenido de $\beta$-caroteno entre genotipos y en la interacción genotipo-ambiente. El contenido de $\beta$-caroteno en los genotipos sembrados en La Molina presentó un rango de 7.32 a $21.32 \mathrm{mg} / 100 \mathrm{~g}$ PF y en y San Ramón de 7.79 a 17.69 mg/100g PF, respectivamente. Mediante el método de los efectos aditivos principales e interacciones multiplicativas (AMMI), se obtuvo una representación gráfica en dos dimensiones (Biplot) como puede apreciarse en la Figura 3. El Biplot identificó a los genotipos 441624 , 440615, 440785, 420081, 440518, 440442 y 440413, como aquellos que mejor expresaron el contenido de $\beta$-caroteno y mejor estabilidad en ambos ambientes (eje de las abscisas). El uso del AMMI permitió una mejor selección de los genotipos para las siguientes etapas de la investigación (cocción y almacenamiento).

Los resultados del contenido de $\beta$ caroteno fueron similares al estudio realizado por van Jaarsveld et al. (2006), quienes obtuvieron un rango de 132 a 194 mg.kg- ${ }^{1}$ PF. Ciertamente, el predominio de color de algunas raíces podría deberse a la concentración del $\beta$-caroteno en toda la raíz. Bajo este criterio, los genotipos 440442 y 440518 , cuyo color predominante fue anaranjado intenso y distribuido homogéneamente en toda la pulpa, presentaron los valores más altos de $\beta$-caroteno en ambos ambientes (Figura 4).

Con respecto a la contribución nutricional y los requerimientos diarios, una porción promedio de $100 \mathrm{~g}$ de camote podría proveer más del $100 \%$ del requerimiento diario 


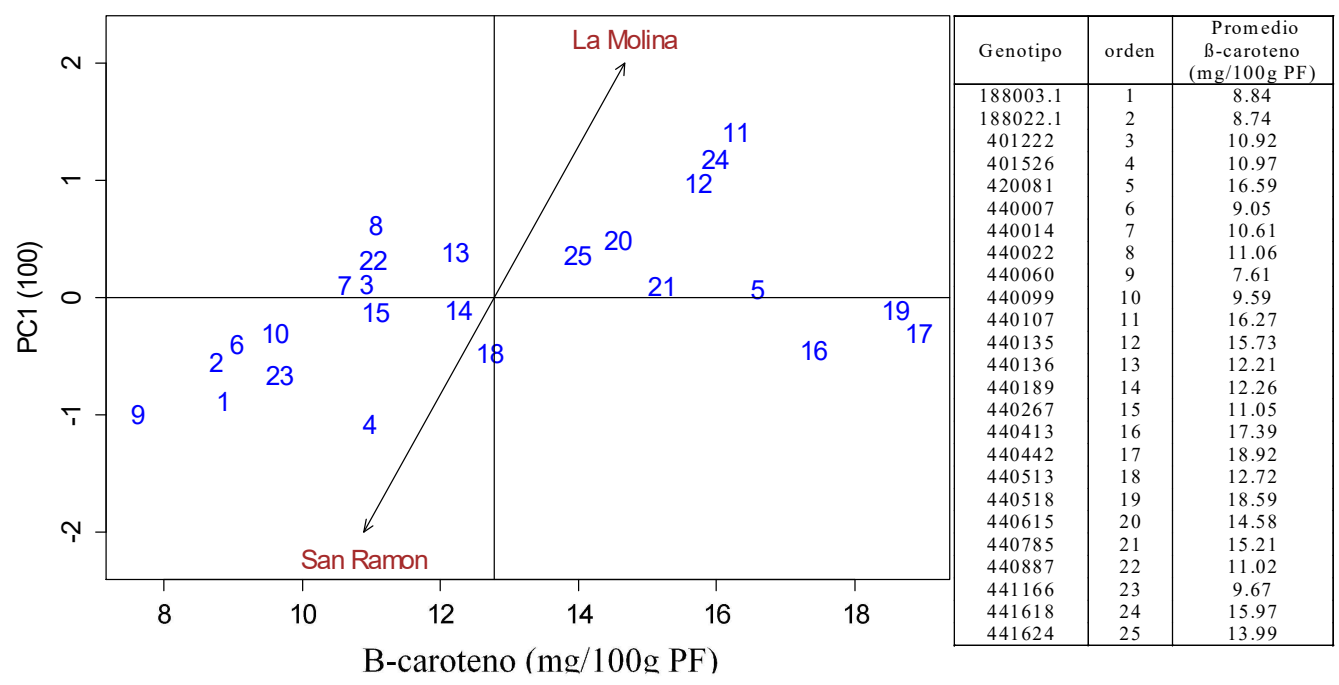

Figura 3. AMMI Biplot para el contenido de $\beta$-caroteno (mg/100 g PF) de 25 genotipos de camote (Ipomoea batatas L) sembrados en La Molina (Lima) y San Ramón (Junín)

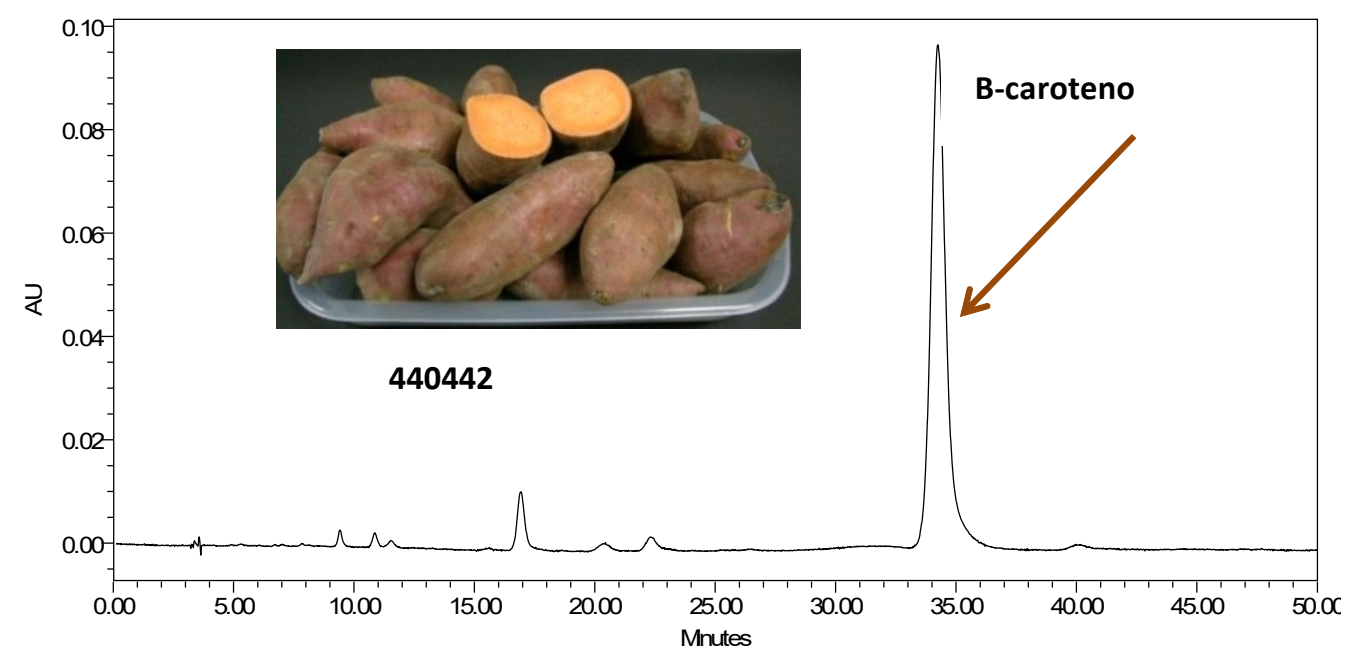

Figura 4. Cromatograma de $\beta$-caroteno del genotipo 440442 (procedente de Taiwán, llamado «Guang Shu 128») de camote (Ipomoea batatas L) obtenido por HPLC 
de $\beta$-caroteno en niños entre 4 a 8 años. Ciertamente, los genotipos 420081, 440413, 440442,440518 con color de pulpa anaranjado pueden proveer más de tres veces el requerimiento diario de contenido de $\beta$-caroteno.

\section{Hierro y Zinc}

Se encontró diferencia significativa $(p<0.05)$ en el contenido de hierro entre genotipos y en la interacción genotipo-ambiente. El contenido de hierro en los genotipos sembrados en La Molina presentó un rango 0.43 a 0.77 mg/100 g PF y en San Ramón de 0.28 a $1.16 \mathrm{mg} / 100 \mathrm{~g}$ PF. La prueba de comparación de promedios indicó que los genotipos 440014 y 188022.1 presentaron los valores más altos de hierro $(0.78$ y $0.96 \mathrm{mg} /$ $100 \mathrm{~g}$ PF) en ambos ambientes. Los resultados del contenido de hierro fueron ligeramente más bajos a los encontrados por Tumwegamire et al. (2011) quienes reportaron un valor promedio de $0.88 \mathrm{mg} / 100 \mathrm{~g} \mathrm{PF}$ en la accesión Carrot Dar, de pulpa anaranjada.

La variación en el contenido de hierro en los genotipos en estudio pudo ser consecuencia de las condiciones propias de cada ambiente. Acorde a los resultados obtenidos, el genotipo 440014 alcanzó una concentración de hierro promedio de $0.78 \mathrm{mg} / 100 \mathrm{~g} \mathrm{PF}$ (Cuadro 1), el cual podría proporcionar alrededor del 16\% de los requerimientos diarios de hierro en niños de edades de 5 a 8 años (10 mg/día) (Food and Nutrition Board, 2011), al consumir una raíz de tamaño comercial de $250 \mathrm{~g}$.

Con respecto al contenido de zinc, se encontró diferencia significativa $(p<0.05)$ entre genotipos; sin embargo, no se encontraron diferencias significativas en la interacción genotipo-ambiente. El contenido de zinc en los genotipos sembrados en La Molina presentó un rango 0.32 a $0.54 \mathrm{mg} / 100 \mathrm{~g}$ PF y en San Ramón de 0.16 a 0.48 mg/100 g PF. La prueba de comparación de promedios encontró que el genotipo 188022.1 presentó el va- lor más alto del contenido de zinc $(0.51 \mathrm{mg} /$ $100 \mathrm{~g}$ PF) en ambos ambientes (Cuadro 1).

La variación en el contenido de zinc en los genotipos por cada ambiente ha podido ser dado por las propias condiciones ambientales. Acorde a los resultados obtenidos, la concentración de zinc en el genotipo 188022.1 podría proveer alrededor el $25 \%$ de los requerimientos diarios de zinc en niños en edades de 5 a 8 años ( $5 \mathrm{mg} /$ día) (Food and Nutrition Board, 2011), al consumir una raíz de tamaño comercial de $250 \mathrm{~g}$.

\section{Cocción sobre el Contenido de $\beta$ - caroteno}

Siete genotipos fueron analizados en crudo y cocido (ebullición y horneado) para evaluar el efecto de la cocción sobre el contenido de $\beta$-caroteno, encontrándose diferencia significativa entre genotipos y métodos de cocción $(p<0.05)$. El tratamiento de cocción por ebullición redujo en menor grado el contenido de $\beta$-caroteno que la cocción por horneado, encontrando diferencias significativas entre los tratamientos (Cuadro 2). La reducción del contenido de $\beta$-caroteno se dio por los cambios oxidativos generados por las altas temperaturas durante la cocción. A temperaturas altas se suelen generar los isómeros cis y la formación de epóxidos, debido a la gran cantidad de dobles enlaces conjugados que presenta el compuesto (Dutta et al., 2006).

El porcentaje de retención del $\beta$ caroteno se encontró en un rango de 77 a $86 \%$ y 56 a $84 \%$ en ebullición y horneado, respectivamente. Similares porcentajes de retención de $\beta$-caroteno en camotes cocidos por ebullido y horneado ( 85 y $73 \%$ ) fueron encontrados por Kidmose et al. (2007) cocinando las raíces con cáscara. Según los resultados, el genotipo 440442 fue el que mejor retuvo el contenido de $\beta$-caroteno después de la cocción. Si bien, los procesos de cocción ocasionaron cierto grado de degradación, 
Cuadro 1. Contenido de materia seca (\%) en 25 genotipos de camote de pulpa anaranjada (Ipomoea batatas L), sembradas en las localidades de San Ramón (Junín) y La Molina (Lima), Perú

\begin{tabular}{|c|c|c|c|c|c|}
\hline $\begin{array}{l}\text { Genotipos } \\
\text { CIP }\end{array}$ & $\begin{array}{c}\text { Nombre de la } \\
\text { accesión }\end{array}$ & $\begin{array}{c}\text { Materia seca } \\
(\%)\end{array}$ & $\begin{array}{c}\beta \text {-caroteno } \\
(\mathrm{mg} / 100 \mathrm{~g} \\
\left.\mathrm{PF}^{\dagger}\right)\end{array}$ & $\begin{array}{c}\text { Hierro } \\
(\mathrm{mg} / 100 \mathrm{~g} \\
\mathrm{PF})\end{array}$ & $\begin{array}{c}\text { Zinc } \\
(\mathrm{mg} / 100 \mathrm{~g} \\
\mathrm{PF})\end{array}$ \\
\hline 440060 & IITA-TIB 4 & $34.07^{\mathrm{a}}$ & $7.62^{j}$ & $0.56^{\text {cdef }}$ & $0.27^{\mathrm{ef}}$ \\
\hline 401526 & $\mathrm{NN}$ & $31.89^{\mathrm{ab}}$ & $10.97^{\text {ghij }}$ & $0.62^{\mathrm{bcd}}$ & $0.41^{\text {abcde }}$ \\
\hline 440513 & Koganesengan & $31.74^{\mathrm{ab}}$ & $12.72^{\text {defgh }}$ & $0.61^{\text {bcde }}$ & $0.37^{\text {bcdef }}$ \\
\hline 401222 & Cuitzo Col. 8463 & $31.16^{\mathrm{ab}}$ & $10.93^{\text {ghij }}$ & $0.49^{\text {def }}$ & $0.33^{\text {cdef }}$ \\
\hline 440007 & W-208 & $30.90^{\mathrm{ab}}$ & $9.05^{\mathrm{ij}}$ & $0.50^{\text {def }}$ & $0.38^{\text {abcdef }}$ \\
\hline 440107 & Sweet Red & $30.07^{\mathrm{abc}}$ & $16.28^{\mathrm{abc}}$ & $0.55^{\text {cdef }}$ & $0.36^{\text {bcdef }}$ \\
\hline 440099 & TIS 9101 & $30.00^{\mathrm{abc}}$ & $9.60^{\mathrm{hij}}$ & $0.58^{\text {cdef }}$ & $0.34^{\text {cdef }}$ \\
\hline 440189 & Tainung No. 64 & $29.21^{\mathrm{abcd}}$ & $12.26^{\text {efghi }}$ & $0.49^{\text {def }}$ & $0.30^{\mathrm{def}}$ \\
\hline 440518 & kyukei No. 34 & $29.07^{\mathrm{abcd}}$ & $18.60^{\mathrm{ab}}$ & $0.45^{\mathrm{def}}$ & $0.30^{\mathrm{def}}$ \\
\hline 440136 & Caromex & $28.83^{\mathrm{abcd}}$ & $12.22^{\text {efghi }}$ & $0.51^{\mathrm{def}}$ & $0.31^{\text {cdef }}$ \\
\hline 188022.1 & UNTACIP & $28.77^{\mathrm{abcd}}$ & $8.74^{\mathrm{ij}}$ & $0.96^{\mathrm{a}}$ & $0.51^{\mathrm{a}}$ \\
\hline 441618 & L9-163 & $28.52^{\mathrm{abcd}}$ & $15.97^{\mathrm{abcd}}$ & $0.46^{\text {def }}$ & $0.30^{\text {def }}$ \\
\hline 188003.1 & $\mathrm{NN}$ & $28.21^{\text {abcde }}$ & $8.84^{\mathrm{ij}}$ & $0.71^{\mathrm{bc}}$ & $0.48^{\mathrm{ab}}$ \\
\hline 440135 & Travis & $28.13^{\text {abcde }}$ & $15.73^{\text {abcde }}$ & $0.60^{\text {bcde }}$ & $0.44^{\mathrm{abc}}$ \\
\hline 441166 & So 268 & $27.85^{\text {bcde }}$ & $9.67^{\mathrm{hij}}$ & $0.48^{\mathrm{def}}$ & $0.29^{\text {def }}$ \\
\hline 420081 & $\mathrm{NN}$ & $27.39^{\text {bcde }}$ & $16.59^{\mathrm{abc}}$ & $0.71^{\mathrm{bc}}$ & $0.37^{\text {bcdef }}$ \\
\hline 440267 & Hung Loc 4 & $27.21^{\text {bcde }}$ & $11.05^{\text {fghij }}$ & $0.58^{\text {cdef }}$ & $0.33^{\text {cdef }}$ \\
\hline 441624 & L $4-13$ & $26.43^{\text {bcdef }}$ & $14.00^{\text {cdefg }}$ & $0.40^{\mathrm{f}}$ & $0.29^{\text {def }}$ \\
\hline 440887 & Ogan & $25.92^{\text {bcdef }}$ & $11.03^{\text {ghij }}$ & $0.45^{\mathrm{def}}$ & $0.28^{\mathrm{def}}$ \\
\hline 440615 & Edd-1 & $24.90^{\text {cdef }}$ & $14.58^{\text {cdef }}$ & $0.53^{\text {cdef }}$ & $0.29^{\text {def }}$ \\
\hline 440014 & $\mathrm{~W}-219$ & $23.63^{\mathrm{def}}$ & $10.61^{\text {ghij }}$ & $0.78^{\mathrm{ab}}$ & $0.34^{\text {cdef }}$ \\
\hline 440413 & Sokol & $23.63^{\mathrm{def}}$ & $17.39^{\mathrm{abc}}$ & $0.51^{\text {def }}$ & $0.29^{\mathrm{def}}$ \\
\hline 440442 & Guang Shu \# 128 & $22.37^{\mathrm{ef}}$ & $18.93^{\mathrm{a}}$ & $0.43^{\text {ef }}$ & $0.28^{\mathrm{ef}}$ \\
\hline 440022 & $\mathrm{~W}-227$ & $21.23^{\mathrm{f}}$ & $11.07^{\text {fghij }}$ & $0.47^{\text {def }}$ & $0.42^{\text {abcd }}$ \\
\hline 440785 & M'bara Delicious & $20.76^{\mathrm{f}}$ & $15.21^{\text {bcde }}$ & $0.41^{\mathrm{f}}$ & $0.25^{\mathrm{f}}$ \\
\hline
\end{tabular}

$a, b, c$ Superíndices distintos representan diferencias significativas entre los promedios $(p<0.05)$. Los resultados representan los promedios de dos repeticiones de campo y de las dos localidades ${ }^{\dagger}$ PF: Peso fresco

el contenido de $\beta$-caroteno en las raíces cocidas de camote (ebullición y horneado) es aun sustancial, pudiendo contribuir significativamente con el requerimiento diario en la dieta de personas saludables.

\section{Contenido de Pro-vitamina A después de la Cocción}

Los resultados de $\beta$-caroteno expresados como pro-vitamina A se muestran en el Cuadro 3. El contenido de $\beta$-caroteno de los 
Cuadro 2. Valores mínimos y máximos del contenido de $\beta$-caroteno (mg/100 g PF) según los tres tipos de cocción: crudo, ebullición y horneado

\begin{tabular}{lccc}
\hline \multirow{2}{*}{$\begin{array}{c}\text { Tratamiento de } \\
\text { cocción }\end{array}$} & \multicolumn{3}{c}{ Contenido de $\beta$-caroteno $(\mathrm{mg} / 100 \mathrm{~g} \mathrm{PF})$} \\
\cline { 2 - 4 } & Valor mínimo & Valor máximo & Promedio \\
\hline $\mathrm{T}_{1}$ : Crudo & 11.38 & 24.72 & $17.22^{\mathrm{a}}$ \\
$\mathrm{T}_{2}$ : Ebullición & 10.29 & 20.52 & $15.15^{\mathrm{b}}$ \\
$\mathrm{T}_{3}$ : Horneado & 9.12 & 19.68 & $14.29^{\mathrm{c}}$ \\
\hline
\end{tabular}

$a, b, c$ Superíndices diferentes en la misma columna representan diferencias significativas $(p<0.05)$

Cuadro 3. Contenido de pro-vitamina A ( $\mu \mathrm{g} \mathrm{RAE} / 100 \mathrm{~g} \mathrm{PF}$ ) en camote (Ipomoea batatas $\mathrm{L}$ ) crudo y cocido por ebullición y horneado, que fuera sembrado en La Molina (Lima)

\begin{tabular}{ccccccc}
\hline \multirow{2}{*}{ Número CIP } & \multicolumn{3}{c}{ Pro-vitamina A $\left(\mu \mathrm{g} \mathrm{RAE} \mathrm{R}^{\dagger} / 100 \mathrm{~g}\right.$ PF $)$} & \multicolumn{3}{c}{$\% \mathrm{RDA}^{\dagger \dagger}$} \\
\cline { 2 - 7 } & Crudo & Ebullición & Horneado & Crudo & Ebullición & Horneado \\
\hline 440442 & $2029^{\mathrm{a}}$ & $1738^{\mathrm{a}}$ & $1706^{\mathrm{a}}$ & $507^{\mathrm{a}}$ & $434^{\mathrm{a}}$ & $427^{\mathrm{a}}$ \\
420081 & $1707^{\mathrm{ab}}$ & $1533^{\mathrm{ab}}$ & $1498^{\mathrm{ab}}$ & $427^{\mathrm{ab}}$ & $383^{\mathrm{ab}}$ & $375^{\mathrm{ab}}$ \\
440413 & $1595^{\mathrm{ab}}$ & $1575^{\mathrm{ab}}$ & $1402^{\mathrm{abc}}$ & $399^{\mathrm{ab}}$ & $393^{\mathrm{ab}}$ & $350^{\mathrm{abc}}$ \\
440135 & $1602^{\mathrm{b}}$ & $1282^{\mathrm{bc}}$ & $956^{\mathrm{cd}}$ & $400^{\mathrm{b}}$ & $320^{\mathrm{bc}}$ & $239^{\mathrm{cd}}$ \\
440615 & $1399^{\mathrm{bc}}$ & $1116^{\mathrm{c}}$ & $1054^{\mathrm{bcd}}$ & $350^{\mathrm{bc}}$ & $279^{\mathrm{c}}$ & $263^{\mathrm{bcd}}$ \\
440014 & $1149^{\mathrm{c}}$ & $1092^{\mathrm{c}}$ & $1039^{\mathrm{d}}$ & $287^{\mathrm{c}}$ & $273^{\mathrm{c}}$ & $260^{\mathrm{d}}$ \\
441166 & $1136^{\mathrm{c}}$ & $1008^{\mathrm{c}}$ & $930^{\mathrm{cd}}$ & $284^{\mathrm{c}}$ & $252^{\mathrm{c}}$ & $233^{\mathrm{cd}}$ \\
\hline
\end{tabular}

a,b,c Superíndices diferentes en la misma columna representan diferencias significativas $(p<0.05)$

'RAE: Actividad equivalente de retinol: $12 \mu \mathrm{g} \beta$-caroteno $=1 \mu \mathrm{g}$ retinol $=1 \mu \mathrm{g}$ RAE (Trumbo et al., 2001)

"RDA: Cantidades diarias recomendadas (Trumbo et al., 2001). Los resultados representan los promedios de dos repeticiones de campo

Cuadro 4. Contenido de $\beta$-caroteno (mg/100 g PF) en siete genotipos de camote (Ipomoea batatas L) sembrados y almacenados en San Ramón (Junín)

\begin{tabular}{cccccccc}
\hline \multirow{2}{*}{$\begin{array}{c}\text { Tratamiento de } \\
\text { almacenamiento } \\
\text { (semanas) }\end{array}$} & 440014 & 440022 & 440413 & 440442 & 440513 & 440615 & $44162^{c}$ \\
\cline { 2 - 8 } & $8.46^{\mathrm{a}}$ & $7.79^{\mathrm{a}}$ & $16.48^{\mathrm{a}}$ & $17.69^{\mathrm{a}}$ & $11.85^{\mathrm{a}}$ & $11.60^{\mathrm{a}}$ & $11.26^{\mathrm{a}}$ \\
\hline 0 & $14.27^{\mathrm{b}}$ & $16.20^{\mathrm{b}}$ & $21.14^{\mathrm{ab}}$ & $24.12^{\mathrm{b}}$ & $15.08^{\mathrm{ab}}$ & $17.40^{\mathrm{b}}$ & $18.19^{\mathrm{b}}$ \\
6 & $16.55^{\mathrm{bc}}$ & $20.33^{\mathrm{bc}}$ & $21.22^{\mathrm{ab}}$ & $30.71^{\mathrm{c}}$ & $17.72^{\mathrm{b}}$ & $20.03^{\mathrm{b}}$ & $23.69^{\mathrm{c}}$ \\
9 & $19.84^{\mathrm{c}}$ & $22.27^{\mathrm{c}}$ & $24.39^{\mathrm{b}}$ & $31.62^{\mathrm{c}}$ & $20.06^{\mathrm{b}}$ & $27.48^{\mathrm{c}}$ & $27.37^{\mathrm{c}}$ \\
\hline
\end{tabular}

$a, b, c$ Superíndices diferentes representan diferencias significativas entre promedios $(p<0.05)$. Los resultados representan los promedios de dos repeticiones de campo 
siete genotipos de camote podría contribuir con el requerimiento diario de $\beta$-caroteno en niños con edades entre 5 a 8 años. Considerando una ingesta promedio de $100 \mathrm{~g}$ de camote de pulpa anaranjada al día, el genotipo 440442 obtuvo una concentración promedio de pro-vitamina A de $1738 \mu \mathrm{g}$ RAE/100 g PF al ser cocidas por ebullición y de $1706 \mu \mathrm{g}$ RAE/100 g PF al ser cocidas por horneado, aportando entre el 427 al $434 \%$ de los requerimientos diarios de $\beta$-caroteno en niños en edades de 5 a 8 años (400 $\mu \mathrm{g} /$ día) (Food and Nutrition Board, 2011). Ciertamente, el potencial de aliviar la deficiencia de vitamina $\mathrm{A}$ mediante el uso de genotipos mejorados de camote de pulpa anaranjada es, hoy en día, uno de los grandes retos en países subdesarrollados.

\section{Almacenamiento sobre el Contenido de $\beta$-caroteno}

El contenido de $\beta$-caroteno en siete genotipos de camote sembrados y almacenados en San Ramón durante 2, 6 y 9 semanas se muestra en el Cuadro 4. Todos los genotipos presentaron diferencias significativas durante el tiempo de almacenamiento $(p<0.05)$. Los genotipos 440413 y 441615 mostraron diferencias no significativas durante la semana 2 y 6 de almacenamiento, en tanto que los genotipos 440442, $440513 \mathrm{y}$ 441624 mostraron diferencias no significativas durante la semana 6 y 9 de almacenamiento.

El contenido de $\beta$-caroteno después de nueve semanas de almacenamiento varió entre 19.84 y $31.62 \mathrm{mg} / 100 \mathrm{~g}$ PF. El genotipo 440442 obtuvo el mayor valor de $\beta$-caroteno (24.12 a $31.62 \mathrm{mg} / 100 \mathrm{~g} \mathrm{PF}$ ).

Las raíces almacenadas de camote concentran en su interior un contenido mayor de vapor de agua, el cual depende enteramente de la temperatura. La diferencia de la concentración de vapor entre al aire del entorno y los espacios intercelulares de la raíz crea una fuerza impulsadora que mueve el vapor de agua desde los espacios más concentrados hacia los menos concentrados, y como existe mayor humedad en los espacios intercelulares, se origina una pérdida de agua de las raíces almacenadas y, posiblemente, cambios en la concentración de carotenoides. En un trabajo previo se demostraron pequeños cambios en el contenido de $\beta$-caroteno y carotenoides totales en raíces almacenadas a 10 y $11^{\circ} \mathrm{C}$ y un incremento cuando se hizo a $20^{\circ} \mathrm{C}$; sin embargo, el incremento no fue tan rápido como a los $15.5^{\circ} \mathrm{C}$, temperatura óptima para la retención de los carotenoides, al menos para los cultivares en estudio (Ezell y Wilcox, 1952). En otro estudio con tres variedades de camote no curados (representando niveles altos, medios y bajos niveles de $\beta$ caroteno) a $13.6{ }^{\circ} \mathrm{C}$, se demostró que la variedad con alta concentración inicial exhibió el mayor incremento del contenido de $\beta$-caroteno después del almacenamiento (Yamamoto y Tomita, 1958).

En general, en el presente estudio, el contenido de $\beta$-caroteno se fue concentrando al incrementar el tiempo de almacenamiento, debido a la evaporación del agua propia de la raíz a causa de la temperatura $\left(15-26^{\circ} \mathrm{C}\right)$ y humedad (44-89.4\%) del medio de almacenamiento.

\section{Conclusiones}

- Se encontró una considerable variación en el contenido de $\beta$-caroteno, hierro y zinc entre los 25 genotipos de camote y entre las localidades de La Molina y San Ramón.

- La variación encontrada debe considerarse en la selección de variedades de camote para los diferentes lugares agrogeográficos.

- Los genotipos 440442 y 420081 presentaron mayor estabilidad del contenido de $\beta$-caroteno durante los procesos de cocción. 
- El genotipo 440442 ( «Guang Shu 128») presentó el más alto contenido de $\beta$ caroteno durante los procesos de cocción (ebullición, horneado) y almacenamiento después de las 9 semanas.

- Las raíces cocidas por ebullición tuvieron una mayor concentración de $\beta$ caroteno que las raíces horneadas.

- La concentración de $\beta$-caroteno fue mayor a medida que aumentó el tiempo de almacenamiento.

\section{Agradecimientos}

Los autores expresan su agradecimiento al programa HarvestPlus por el soporte financiero para hacer posible el presente trabajo. Asimismo, al Ing. Víctor Suárez del Centro Internacional de la Papa (CIP) por su apoyo en el análisis de los datos.

\section{Literatura Citada}

1. Andrade M, Barker I, Cole D, Dapaah $H$, Elliott $H$, Fuentes $S$, Grüneberg $W$, et al. 2009. Unleashing the potential of sweetpotato in SubSaharan Africa: current challenges and way forward. International Potato Center (CIP). Social Sciences Working Paper 2009-1. ISSN 0256-8748. 197 p. doi: 10.4160/0256874820091

2. Bechoff A, Tomlins K, Dhuique-Mayer C, Dove R, Westby A. 2011. On-farm evaluation of the impact of drying and storage on the carotenoid content of orange-fleshed sweet potato (Ipomea batata Lam). Int J Food Sci Tech 46: 52-60. doi:10.1111/j.1365-2621.2010.02450.x

3. Bonierbale M, Burgos G, zum Felde T, Sosa P. 2010. Composition nutritionnelle des pommes de terre. Cahiers de Nutrition et de Diététique 45(6): S28-S36. doi: 10.1016/S00079960(10)70005-5
4. Dutta D, Dutta A., Raychaudhuri U, Chakraborty R. 2006. Rheological characteristics and thermal degradation kinetics of â-carotene in pumpkin puree. J Food Eng 76: 538-546. doi: 10.1016/ j.jfoodeng.2005.05.056

5. Ezell BD, Wilcox MS, Crowder JN. 1952. Pre- and post-harvest changes in carotene, total carotenoids and ascorbic acid content of sweetpotato. Plant Physiol 27: 355-369.

6. $\mathrm{FAO/WHO}$. 2002. Vitamin A in human vitamin and mineral requirements. Report of joint FAO/WHO expert consultation Bangkok, Thailand. 286 p. [Internet]. Disponible en: http://www.fao.org/3/ay2809e.pdf

7. Food and Nutrition Board. 2011. Dietary reference intakes for vitamin a, vitamin $\mathrm{k}$, arsenic, boron, chromium, copper, iodine, iron, manganese, molybdenum, nickel, silicon, vanadium, and zinc. Washington DC: National Academy Press. 773 p.

8. García-Casal MN, Layrisse M, Solano L, Barón MA, Arguello F, Llovera D, Ramírez J, et al. 1998. Vitamin A and beta-carotene can improve non-heme iron absorption from rice, wheat and corn by humans. J Nutr 128: 646-650.

9. Hansen TH, Laursen KH, Persson, DP, Pedas, P, Husted S, Schjoerring JK. 2009. Micro-scaled high-throughput digestion of plant tissue samples for multielemental analysis. Plant Methods 5(12). doi: 10.1186/1746-4811-5-12

10. [INIA] Instituto Nacional de Innovacion Agraria. s.f. Centro Experimental La Molina - Ubicación Geográfica. [Internet]. Disponible en: http://www.inia.gob.pe/la-molina/ ubicacionmolina

11. Kapinga RE, Ewell PT, Jeremiah SC, Kileo R. 2007. Sweetpotato in Tanzania farming and food systems: implications for research. International Potato Center (CIP)/Ministry of Agriculture, Tanzania. 47 p. [Internet]. Disponible en: http:// pdf.usaid.gov/pdf_docs/PNABY619.pdf 
12. Kidmose U, Christensen LP, Agili SM, Thilsted SH. 2007. Effect of home preparation practices on the content of provitamin A carotenoids in colored sweetpotato varieties (Ipomoea batatas Lam.) from Kenya. Innov Food Sc Emerg Technol 8: 399-406. doi: 10.1016/ j.ifset.2007.03.025

13. Moraghan JT, Mascagni Jr HJ. 1991. Environmental and soil factors affecting micronutrient deficiencies and toxicities. In: Mordvedt JJ, Cox FR, Shumann LM, Welch RM (eds). Micronutrients in agriculture. Madison, WI, USA: Soil Science Society of America. p 371-425.

14. Porras E, Burgos G, Sosa P, zum Felde T. 2014. Procedures for sampling and sample preparation of sweetpotato roots and potato tubers for mineral analysis. Lima, Peru: International Potato Center (CIP). 13 p. doi: 10.4160// 9789290604457

15. Reynel C, León J. 1989. Especies forestales de los bosques secundarios de Chanchamayo. Proyecto de utilización de bosques secundarios en el trópico húmedo peruano. Lima: Universidad Nacional Agraria La Malina. $173 \mathrm{p}$.

16. Rodriguez-Amaya DB, Kimura M. 2004. HarvestPlus handbook for carotenoid analysis. Washington DC: HarvestPlus. 63 p.

17. Shapiro SS, Wilk MB. 1965. An analysis of variance test for normality (complete samples). Biometrika 52: 591-611.

18. Tiempo Agrario. 2010. El camote se abre paso en San Ramón. [Internet]. Disponible en: http://tiempoagrario.blogspot.pe/2010/09/el-camote-se-abrepaso-en-san-ramon.html
19. Trumbo P, Yates AA, Schlicker S, Poos M. 2001. Dietary reference intakes: vitamin A, vitamin $\mathrm{K}$, arsenic, boron, chromium, copper, iodine, iron, manganese, molybdenum, nickel, silicon, vanadium, and zinc. J Am Diet Assoc 101: 294-301. doi: 10.1016/S00028223(01)00078-5

20. Tumwegamire S. 2011. Genetic variation, diversity and genotype by environment interactions of nutritional quality traits in East African sweetpotato. PhD Thesis. Kampala, Uganda: Makerere University. 34 p.

21. Tumwegamire $S$, Kapinga $R$, Rubaihayo P, LaBonte D, Grüneberg W, Burgos G, Zum Felde T, et al. 2011. Evaluation of dry matter, protein, starch, sucrose, $\beta$-carotene, iron, zinc, calcium, and magnesium in East African sweetpotato [Ipomoea batatas (L.) Lam] germplasm. HortScience 46: 348-357.

22. van Jaarsveld PJ, De Wet M, Harmse $E$, Nestel P Rodriguez-Amaya DB. 2006. Retention of $\beta$-carotene in boiled, mashed orange-fleshed sweet potato. J Food Comp Anal 19: 321-329. doi: 10.1016/j.jfca.2004.10.007

23. Wang XD. 1994. Review: absorption and metabolism of beta-carotene. J Am Coll Nutr 13: 314-325. doi: 10.1080/ 07315724.1994.10718416

24. Woolfe JA. 1992. Sweet potato: an untapped food resource. UK: Cambridge University Press. 643 p.

25. Yamamoto Y, Tomita Y. 1958. Studies on the bio-pigments and vitamins: IV. Correlative changes in the carotene, total carotenoids and the other constituents of sweet potatoes during storage (1). Mem Fac Agric, Kagosbima Univ 3(2): 63-68. 